\title{
Surgical Management of Acute Pulmonary Thromboembolism: our Experience
}

\author{
Chaitanya Raut, Prashant Mishra, Chandan Kumar Ray Mohapatra, Jayant. V. \\ Khandekar, Harsh Sateesh Seth, Ganesh Kumar K. Ammannaya \\ Department Of Cardiovascular And Thoracic Surgery, Lokmanya Tilak Municipal Medical College And \\ General Hospital Mumbai, India
}

\begin{abstract}
Acute pulmonary thromboembolism (PTE) can be a life threatening complication of deep vein thrombosis. Surgical embolectomy is a rarely performed and is only reserved for hemodynamically unstable patients, those with massive pulmonary thromboembolism and those with failed thrombolysis therapy. We reviewed the results of surgical pulmonary embolectomy performed in our institute over the last four years. Data was retrospectively studied of 28 patients who underwent pulmonary embolectomy in our institute over the last four years i.e. from 2012 to 2016. Demographic data, clinical presentation and outcomes were analyzed. Clinical presentation included dyspnea (82\%), hemodynamic instability requiring continuous vasopressor support (36\%), echocardiographic evidence of right ventricular dilatation (82\%). There was $3 \%$ mortality rate in our study which is comparably low with previous studies. Our series implies the benefit of early consideration and performance of pulmonary embolectomy in these critically ill patients.
\end{abstract}

keywords: Pulmonary embolectomy, echocardiogram , pulmonary thromboembolism(PTE)

\section{Introduction}

Acute pulmonary thromboembolism (PTE) can be a life threatening complication of deep vein thrombosis. Standard treatment options include anticoagulation, systemic fibrinolytic therapy; catheter based thrombolytic therapy and surgical pulmonary embolectomy ${ }^{1}$. Surgical embolectomy is a rarely performed and is only reserved for hemodynamically unstable patients, those with massive pulmonary thromboembolism and those with failed thrombolysis therapy ${ }^{2}$. The procedure itself is traditionally associated with mortality rate of 9 to $30 \%^{3-6}$. We reviewed the results of surgical pulmonary embolectomy performed in our institute over the last four years. This study also explored the need for expanding the indications of surgical embolectomy.

\section{Material and Methods}

Data was retrospectively studied of 28 patients who underwent pulmonary embolectomy in our institute over the last four years i.e. from 2012 to 2016. The pre-operative data included: age, sex, symptoms during first presentation, pre-operative circulatory shock, history of DVT or previous thromboembolism and history of thrombolysis received. Pre-operative lower limb venous Doppler, 2D echo and CT pulmonary angiography findings were noted.

The details of surgical technique were recorded. All patients underwent surgery using cardiopulmonary bypass with aortic and bicavalcannulation. Moderate hypothermia with aortic cross clamping and cardioplegic arrest was used in 25 patients. Three patients required hypothermia with circulatory arrest. The technique of surgery involved separate incision for right and left pulmonary artery for thrombus removal. Post-operative in hospital mortality, ICU stay, neurological event, renal dysfunction, platelet count, elevation of transaminase and time to extubation was documented. Post-operative CT was done in all patients before discharge. CT findings and medications at the time of discharge were also noted. Clinical data at one month follow up was collected.

\section{Results}

From February 2012 to May 2016 a total of 28 patients: 25 male (89\%) and 3 females (11\%) were operated for acute pulmonary thromboembolism. The mean age of the patients was 51 years (range 41 to 68 years). The patient characteristics are shown in Table 1.

Table 1: Patient demographics

\begin{tabular}{|l|l|}
\hline Characteristics & \\
\hline Age & 41 to 68 years (mean 51) \\
\hline Female patients & $3(10 \%)$ \\
\hline Onset of pulmonary embolism during hospital admission & $7(25 \%)$ \\
\hline Previous admission for PTE & $3(10 \%)$ \\
\hline
\end{tabular}


Twenty three patients $(82 \%)$ presented with dyspnea at rest, $12(42 \%)$ presented with retrosternal chest pain. The other presenting symptoms are shown in Table 2

Table 2: Symptoms at presentation

\begin{tabular}{|l|l|}
\hline Symptoms & $\mathrm{n}(\%)$ \\
\hline Dyspnea at rest & $23(82)$ \\
\hline Retrosternal chest pain & $12(43)$ \\
\hline Syncope & $2(7)$ \\
\hline Hemoptysis & $5(18)$ \\
\hline Shock & $10(36)$ \\
\hline Leg edema & $4(14)$ \\
\hline
\end{tabular}

Ten patients $(46 \%)$ were hemodynamically unstable presenting with circulatory collapse. Four patients (14\%) had clinical evidence of deep vein thrombosis in the form of pedal edema. However on venous color Doppler scan $25(89 \%)$ patients had evidence of deep vein thrombosis. Three patients did not show any evidence of deep vein thrombosis. Three patients gave history of previous pulmonary thromboembolism (treated non operatively). CT pulmonary angiography was performed in all patients and the confirmatory diagnosis was made in them using this modality. Two dimensional echocardiography (2D echo) was also done in all patients. Twenty four $(85 \%)$ patients showed dilatation of RV and RV pressure overload pattern. Two patients also had evidence of right atrial thrombus detected by $2 \mathrm{D}$ echo. Importantly 18 patients had received systemic thrombolysis treatment but did not recover and were taken up for surgery

The investigational characteristics before surgery are shown in Table 3

Table 3: Preoperative 2D echo and CT pulmonary angiography findings

\begin{tabular}{|l|l|}
\hline Findings & $\mathrm{n}, \%$ \\
\hline Embolisation in large central branch* & $16(57)$ \\
\hline Free Right atrial thrombosis & $2(7)$ \\
\hline Failed thrombolysis & $18(64)$ \\
\hline Dilatation of RV & $23(82)$ \\
\hline
\end{tabular}

*Main, right and left pulmonary artery

Cardiopulmonary bypass was used in all patients. CPB time was ranging from 42 minutes to 79 minutes; $\quad($ Mean $\pm 2 \mathrm{SD}=59 \pm 13.6)$. Four patients required deep hypothermia circulatory arrest. One patient died post-operatively day five due to multiorgan failure. Two patients developedtransient renal dysfunction which manifested in the form of raised serum creatinine, which recovered before hospital discharge. No patient developed neurological dysfunction. Time to extubation from the time to shifting from OT was on an average 15 hours. Three patients required prolonged ventilation ( $>48$ hours). Average ICU stay was 5.4 days $( \pm 3.3)$. The operative and post-operative findings are shown Table 4

Table 4: Intra-operative and post-operative data

\begin{tabular}{|l|l|}
\hline Parameter & \\
\hline CPB time & $59 \pm 13.6$ minutes \\
\hline DHCA & 4 \\
\hline Moratality & $1(3 \%)$ \\
\hline Renal dysfunction & $2(6 \%)$ \\
\hline Neurological damage & 0 \\
\hline Thrombocytopenia & 12 \\
\hline Raised Transaminases & 10 \\
\hline Time to extubation & $15 \pm 6.8$ hours \\
\hline ICU stay & $5.4 \pm 3.3$ days \\
\hline
\end{tabular}

Post operatively 27 patients underwent CT pulmonary angiography before discharge. Twenty four patients showed complete resolution of the thrombus from the main right and left pulmonary artery and also the lobar branches. Two patients showed thrombosis with partial lumen occlusion of the right descending pulmonary artery. One patient had thrombosis of bilateral $(\mathrm{L}>\mathrm{R})$ lobar and segmental branches but without any luminal compromise.

All patients were started on oral anticoagulation with target INR of range 2 to 3 . Three patients were receiving lifelong anticoagulant treatment. Twenty three patients were symptom free at 1 month follow up. IVC filter was inserted in 3 patients who had recurrence of PE. 
Pumonarythromboembolectomy is one of the oldest performed cardiac surgeries ${ }^{7-8}$. However due to high mortality it was rarely performed even with the advent of cardiopulmonary bypass ${ }^{9}$. Recent data suggest that there has been a gradual increase in the number of pulmonary embolectomies performed ${ }^{10}$. Traditional indications for embolectomy include massive embolization, hemodynamically unstable patient with pulmonary embolism and failed thrombolysis therapy ${ }^{2}$. Acute pulmonary embolism can present with a variety of clinical features. Patients usually have preceding history of deep vein thrombosis. Even in our study $78 \%$ patients had evidence of DVT. Schwartzet $\mathrm{al}^{11}$ demonstrated that $6 \%$ of patients out of 230 had no evidence of deep vein thrombosis. In our study the number was high (10\%) but that could be because of limited number of patients and upper limb Doppler not performed in this subset. Importantly only $14 \%$ of patients had clinical evidence of deep vein thrombosis. This highlights the importance of performing Doppler scan of the lower limb in all patients. Dyspnea at rest is the most common clinical finding followed by chest pain, hemoptysis and syncope. Ten patients (46\%) presented with circulatory collapse and shock. They were shifted to OT immediately after the diagnosis was made on CT pulmonary angiography. Ikuoet al ${ }^{12}$ demonstrated the importance of prompt surgical embolectomy in the critically ill patients. They reported operative mortality of $5.3 \%$ in the critically ill patients.

CT pulmonary angiography is rapid and accurate diagnostic test for suspected pulmonary embolism. Kristoferet $\mathrm{al}^{13}$ showed that CT pulmonary angiography can be performed within minutes even in hemodynamically unstable patient. The definitions of massive (high risk), sub massive (intermediate risk) and non-massive (low risk) embolism have been given by Reed et $\mathrm{al}^{14}$. the abbreviated form of their definitions are given in Table 5

Table 5: Clinical Features of Low, Intermediate, and High-Risk Pulmonary Embolism

\begin{tabular}{|l|l|l|l|}
\hline Findings & $\begin{array}{l}\text { Low risk } \\
\text { (non massive) }\end{array}$ & $\begin{array}{l}\text { Intermediate risk } \\
\text { (sub-massive) }\end{array}$ & $\begin{array}{l}\text { High risk } \\
\text { (massive) }\end{array}$ \\
\hline Hemodynamic status & normal & normal & unstable \\
\hline hypoxia & may be present & likely present & present \\
\hline RV dysfunction & normal & likely present & present \\
\hline Serum troponin & normal & likely elevated & elevated \\
\hline
\end{tabular}

In our institute patients with massive pulmonary embolization and/or hemodynamically unstable patients were taken up for emergency embolectomy. However there was another subgroup of patients with sub massiveembolization, stable hemodynamics and 2D echo evidence of dilated RV who were operated. These were the patients who failed to show resolution of symptoms after thrombolytic therapy. These intermediate risk group patients are at a high risk of subsequent massive embolism. Khalid et al ${ }^{15}$ also demonstrated that thrombolysis for sub massive embolism carries higher death rate and increased risk of major hemorrhage and increased risk of recurrent embolism. Neely et $\mathrm{al}^{16}$ operated 56 patients with sub massive embolism becauseof contraindications to fibrinolysis, failure of catheter-based therapies,or severe RV dysfunction. In the subgroupof patients with submassive PE,the operative mortality rate was 3.6\%. The mortality rate in our series was only $3 \%$. The only death which occurred was due to multiorgan failure. He was diagnosed to have hepatocellular carcinoma on post mortem examination. There were no deaths in the one month follow up period. The mortality rate of surgical embolectomy has been gradually decreasing over the last few decades. Satoshi et al reported operative mortality of $18.8 \%$ whereas Leacche Met $\mathrm{al}^{3}$ reported operative mortality of $6 \%$ and late mortality of $12 \%$. Neely et $\mathrm{al}^{16}$ also demonstrated overall mortality of $6.6 \%$.

Our average CPB tine was $59 \pm 13.6$ minutes. This was not studied in any of the previous reports. However it was far less than the average safe CPB time reported with respect to other cardiac surgeries ${ }^{17}$. The average ICU stay was $(5.4 \pm 3.3$ days $)$ in our study. This was comparable to the average ICU stay reported by Fukuda et $\mathrm{al}^{12}(4.2 \pm 5.0$ days). There was no neurological event in our study. The renal dysfunction, thrombocytopenia and raised transaminases were all transient and found to be normal at the time of discharge in all patients. Only 3 patients had residual thrombosis in the post-operative CT scan. All of them were managed conservatively as none of them had luminal compromise. Post operatively all patients were started on oral anticoagulation for a therapeutic INR of 2 to 3 . The duration of anticoagulation therapy was tapered to individual patients. Overall 3 patients with history of recurrent pulmonary thromboembolism and other three having persistent risk factors were found to be on lifelong anticoagulation treatment.

The role of IVC filter in PTE is still a topic of debate. S Gupta et al ${ }^{18}$ advocated IVC filter in patients where anticoagulation is either contraindicated or fails to prevent recurrent PE. Kucheret al ${ }^{19}$ had found that none of the 11 patients who received an IVC filter developed recurrent PE within 90 days, and 10 (90.9\%) survived at least 90 days. However late complicationsare more frequent and include recurrent DVT inapproximately $13 \%$ to $44 \%$ of patients ${ }^{20-22}$.

was collected retrospectively. The long term data of recently operated patients is yet to be observed. The data for biochemical markers was not available and hence not presented. 


\section{VI.Conclusion}

Surgical pulmonary embolectomy is a lifesaving procedure for patients with massive PTE. Even those with sub massive thrombosis and failed thrombolytic therapy can benefit from surgery. Surgical embolectomy is a safe procedure and can be performed effectively at centers having CPB facilities. The operative mortality of this surgery has significantly reduced over the last few decades. The role of anticoagulation has been firmly established, however the necessity for lifelong anticoagulation needs to be investigated. Similarly the indications of IVC filter require further studies.

\section{References}

[1]. 2014 ESC Guidelines on the diagnosis andmanagement of acute pulmonary embolismdoi:10.1093/eurheartj/ehu283

[2]. Guidelines for the Diagnosis, Treatment andPrevention of Pulmonary Thromboembolism andDeep Vein Thrombosis (JCS 2009)

[3]. Circ J 2011; 75: $1258-1281$

[4]. Leacche $\mathrm{M}^{1}$, Unic D, Goldhaber SZ, et al.Modern surgical treatment of massive pulmonary embolism: results in 47 consecutive patients after rapid diagnosis and aggressive surgical approach.

[5]. J ThoracCardiovasc Surg. 2005 May; 129(5):1018-23

[6]. Satoshi Taniguchi, Wakako Fukuda, Ikuo Fukuda, et al. Outcome of pulmonary embolectomy for acute pulmonary thromboembolism: analysis of 32 patients from a multicentre registry in Japan InteractCardiovascThorac Surg. 2012 Jan; 14(1): 6467

[7]. LishanAklog, Christopher S. Williams, John G. Byrne et al.Acute Pulmonary EmbolectomyA Contemporary Approach Circulation 2002;105:1416-1419

[8]. Timothy J. Poterucha, MD; Brian Bergmark, MD; SaryAranki, MD; et al. Surgical Pulmonary Embolectomy CirculationAHA.115.015916

[9]. Kirschner M[Eindurch die Trendelenburgsche Operation geheilten Fall von Embolie der Art.Pulmonalis Arch Klin Chir 1924;133:312-59.

[10]. McFadden PM, Ochsner JL. Aggressiveapproach to pulmonary embolectomy formassive acute pulmonary embolism: a historicaland contemporary perspective. MayoClin Proc. 2010;85:782-784.

[11]. Oakley CM et alThere is no place for acute pulmonary embolectomy. Br J Hosp Med 1989;41:469

[12]. Gulba DC, Schmid C, BorstHGet al. Medical compared withsurgical treatment for massive pulmonaryembolism Lancet. 1994;343:576-577

[13]. Schwartz T1, Hingorani A, Ascher E et al.Pulmonary embolism without deep venous thrombosis. Ann Vasc Surg. 2012 Oct;26(7):973-6.

[14]. Ikuo Fukuda, MD, Satoshi Taniguchi, MD, Kozo Fukui et al. Improved Outcome of Surgical Pulmonary Embolectomy by Aggressive Intervention for Critically III Patients Ann Thorac Surg. 2011 Mar;91(3):728-32

[15]. Kristofer M Schramm, MD Acute Pulmonary Embolism (Helical CT)

[16]. Grant W Reed, MD; Mehdi H. Shishehbor et al.Contemporary Management of Acute Pulmonary Embolism: A Focus on Intermediate Risk Patients Latest in cardiology: American cardiology society; March 3`1 2015

[17]. 15] Khalid AlmoosaIs Thrombolytic Therapy Effectivefor Pulmonary Embolism?

[18]. Am Fam Physician 2002;65:1097-102

[19]. Neely RC, Byrne JG, Gosev I et alSurgical Embolectomy for Acute Massive and Submassive Pulmonary Embolism in a Series of 115 Patients Ann Thorac Surg. 2015 Oct;100(4):1245-51

[20]. Nissinen J1, Biancari F, Wistbacka JO et al. Safe time limits of aortic cross-clamping and cardiopulmonary bypass in adult cardiac surgery. Perfusion 2009 Sep;24(5):297-305

[21]. S Gupta, Brig MM Gupta. Acute Pulmonary Embolism Advances in Treatment JAPImarch2008;56:185-91

[22]. Nils Kucher, Elisa Rossi, Marisa De Rosa et al. Massive Pulmonary Embolism CirculationAHA.105.592592

[23]. Blebea J, Wilson R, Waybill P, et al. Deep venous thrombosis after percutaneous insertion of vena caval filters. J Vasc Surg. 1999;30:821-829

[24]. Greenfield L J, Proctor M C. Recurrent thromboembolism in patients with vena cava filters.

[25]. J Vasc Surg. 2001;33:510-514

[26]. Wojcik R, Cipolle M D, Fearen I, et al. Long term follow-up of trauma patients with a vena caval filter J Trauma 2000;49:839-843 